\title{
Focus optimization of relativistic self-focusing for anomalous laser penetration into overdense plasmas (super-penetration)
}

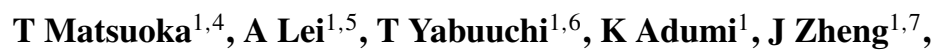 \\ R Kodama ${ }^{1}$, K Sawai ${ }^{2}$, K Suzuki ${ }^{2}$, Y Kitagawa ${ }^{2,8}$, T Norimatsu ${ }^{2}$,

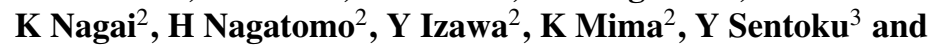 \\ K A Tanaka ${ }^{1}$ \\ ${ }^{1}$ Graduate School of Engineering and Institute of Laser Engineering, Osaka University, \\ 2-1 Yamada-oka, Suita, Osaka 565-0871, Japan \\ ${ }^{2}$ Institute of Laser Engineering, Osaka University, 2-6 Yamada-oka, Suita, Osaka 565-0871, \\ Japan \\ ${ }^{3}$ University of Nevada, Reno, Department of Physics, NTF-MS372, 5625 Fox Avenue, Reno, \\ NV 89506, USA
}

Received 5 May 2008, in final form 24 July 2008

Published 10 September 2008

Online at stacks.iop.org/PPCF/50/105011

\begin{abstract}
Relativistic electron motion in a plasma due to an intense laser pulse modifies the refractive index and leads to two effects: relativistic induced transparency and relativistic self-focusing. A combination of the above two effects enables transmission of laser energy deep into plasmas which is useful for fast ignition of inertial fusion. This so-called super-penetration sensitively depends on the focal position of the laser intensity due to the inhomogeneous density profile of the plasma and convergence of the laser pulse by final focusing optics. Experiments were conducted at vacuum focused laser intensities between 3.3 and $4.4 \times 10^{18} \mathrm{~W} \mathrm{~cm}^{-2}$ at peak plasma densities between 23 and $75 n_{\mathrm{c}}$, where $n_{\mathrm{c}}$ is the critical density of the plasma. We introduced a scenario: the laser beam diameter at $n_{\mathrm{c}} / 4$ density must be smaller than the plasma wavelength to achieve whole beam self-focusing. An optimum focus was found experimentally by measuring the plasma channel, laser transmittance and electron spectra.
\end{abstract}

\footnotetext{
${ }^{4}$ Present address: FOCUS Center and Center for Ultrafast Optical Science, University of Michigan, Ann Arbor, MI 48109 USA.

${ }^{5}$ Present address: Shanghai Institute of Optics and Fine Mechanics, Shanghai, 201800, People's Republic of China.

6 Present address: University of California, San Diego, CA 92093, USA.

${ }^{7}$ Present address: University of Science and Technology of China, Hefei, 230026, People's Republic of China.

${ }^{8}$ Present address: The Graduate School for the Creation of New Photonics Industries, Kurematsu-cho, 1955-1, Nakaku, Hamamatsu 431-202, Japan.
} 
All three data are consistent with one another and numerical calculations based on a paraxial approximation model suggest that this optimum focus corresponds to the scenario described above.

(Some figures in this article are in colour only in the electronic version)

\section{Introduction}

Anomalous transmission due to relativistic induced transparency (RIT) [1] and hole boring (HB) [2,3] have been studied for applications in fast ignition (FI) for inertial fusion. The FI scheme requires a pre-compressed dense plasma core which is then heated by an ultra-intense laser pulse called a heating pulse [4]. The laser pulse will be reflected and absorbed at the critical density of the plasma, given by $n_{\mathrm{c}}=10^{21} \mathrm{~cm}^{-3}$ for a laser wavelength of $1.053 \mu \mathrm{m}$ in the non-relativistic limit. The critical density is approximately five orders of magnitude less than the compressed core density. Relativistic electron beams generated by the heating pulse transport energy into the core. The electron beam is mainly generated near the critical density and has a certain divergence angle. Based on the divergence of the electron beam and the distance between the critical density and the core, the electron beam may overfill the core, decreasing heating efficiency. Therefore, minimizing the distance between the critical density and the core can increase heating efficiency in the FI scheme.

Two methods have been proposed so far to minimize the distance: cone guiding [5] and plasma channeling [4]. A gold cone is inserted into the fuel shell such that the tip is placed slightly away (about $50 \mu \mathrm{m}$ ) from the center of the shell. The cone shields plasma from the compression pulse in order to allow the heating pulse to propagate freely within the cone. Therefore, electrons generated at the tip of the cone only have to propagate a short distance to reach the core. This method successfully enhanced the thermal neutron yield [5]. However, this target geometry does not allow the symmetrical laser compression necessary in high gain designs and has to be tested with a DT fuel shell. In addition, the cone debris could be a potential threat to the final optics in future laser fusion reactors.

Plasma channeling allows for a symmetrical implosion, in contrast to the cone scheme. An ultra-intense laser pulse prior to the heating pulse opens a channel due to plasma cavitation making use of the ponderomotive force of the laser [2,3]. The channeling is well studied for underdense plasmas where the electron density is less than the critical density. In a previous work, the transmittance of two sub-picosecond laser pulses co-propagating in a uniform underdense plasma showed an increase from a few per cent up to $90 \%$ by changing the delay between the pulses [6]. Kodama et al studied channel formation by $100 \mathrm{ps}$ laser pulses in overdense plasmas. The ponderomotive force of the laser creates a channel due to the HB process but the length is limited by ion dynamics [2,3]. Alternatively, laser pulses exceeding a focused intensity of $10^{18} \mathrm{~W} \mathrm{~cm}^{-2}$ can propagate into overdense plasma due to RIT and the accessible density is larger for higher intensity. On the other hand, relativistic self-focusing (RSF) could guide a laser pulse without diffracting for a long distance and can focus the laser pulse down to a spot diameter of roughly its wavelength when the power is greater than a critical power: $P_{\mathrm{RSF}}=17 n_{\mathrm{c}} / n_{\mathrm{e}}(\mathrm{GW})$ at a plasma density of $n_{\mathrm{e}}[7]$.

Combining RSF and RIT (super-penetration) allows efficient transport of laser pulse energy toward the core. Tanaka et al demonstrated effective energy transfer by a subpicosecond laser at $100 \mathrm{TW}$ laser power through a preformed plasma generated by separate laser pulses [8,9] and proposed super-penetration. Strong X-ray emission was observed at the target surface, which indicates that the laser could propagate $100 \mu \mathrm{m}$ in overdense inhomogeneous 

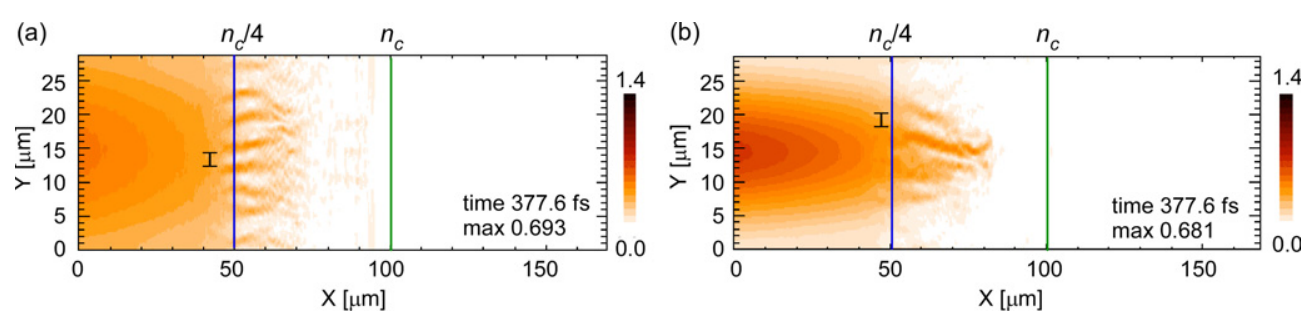

Figure 1. Contour images of the square root of laser intensity for $(a)$ large diameter and $(b)$ small diameter laser beam. A $5 \mu \mathrm{m}$ FWHM (full width at half maximum) focal spot diameter of the laser pulse at $\lambda=1 \mu \mathrm{m}$ was set at (a) $X=150 \mu \mathrm{m}$ and (b) $X=50 \mu \mathrm{m}$. A Gaussian beam was assumed in order to simulate a converging laser beam. The temporal intensity profile is Gaussian with $495 \mathrm{fs}$ FWHM. The peak intensity of the laser in vacuum at focus was set at $10^{19} \mathrm{~W} \mathrm{~cm}^{-2}$. Polarization of the laser is linear and in the $X-Y$ plane. Plasma density linearly increases from $0.05 n_{\mathrm{c}}$ at $X \sim 36.7 \mu \mathrm{m}$ to $n_{\mathrm{c}}$ at $X \sim 100 \mu \mathrm{m}$. The locations of $n_{\mathrm{c}} / 4$ and $n_{\mathrm{c}}$ are indicated by vertical lines, respectively. The scale bar in the images shows the length of $\lambda_{\mathrm{p}} \sim 2 \mu \mathrm{m}$ in the $\mathrm{Y}$ direction. The laser propagates from left to right. Note that the scale of $X$ and $Y$ direction is different.

plasmas up to solid density by adjusting the focal position of the laser relative to the plasma. This work indicated that the penetration capability can be controlled by adjusting the focal position relative to the plasma which controls RSF and RIT.

The heating pulse might suffer from the filamentation instability triggered by stimulated Raman scattering (SRS). Once the laser pulse breaks into many filaments, the intensity of each filament is reduced by the number of filaments and penetration capability due to RIT is significantly limited. Those filaments could merge together in a later time of interaction [10] but controllability of the merging process is questionable thus far. Therefore, minimizing filamentation instability is key for super-penetration. The SRS growth rate is maximized at one-quarter critical density $\left(n_{\mathrm{c}} / 4\right)$ in the non-relativistic theory [11]. Figure 1 shows square root intensity contour images which were obtained by 2D particle-in-cell (PIC) simulations from the PICLES2D [12]. The focal position of the laser was adjusted in order to obtain a large $(10 \mu \mathrm{m})$ and a small $(5 \mu \mathrm{m})$ spot diameter at $n_{\mathrm{c}} / 4$. Other simulation parameters are given in the caption of figure 1. Many filaments are observed for the large spot diameter simulation but few filaments are observed for a small diameter spot simulation as shown in figure 1 . The separation between filaments is equal to the plasma wavelength $\left(\lambda_{\mathrm{p}} \sim 2 \mu \mathrm{m}\right)$ at $n_{\mathrm{c}} / 4$. The smaller spot diameter clearly reduces the number of filaments. Both simulations show that the filamentation starts from $n_{\mathrm{c}} / 4$. Therefore, making the laser spot diameter smaller than $\lambda_{\mathrm{p}}$ at $n_{\mathrm{c}} / 4$ could reduce the filamentation instability significantly and could promote superpenetration.

In this paper, we studied propagation behavior of a converging laser pulse in an inhomogeneous plasma with experiments and numerical calculations. The laser propagation was controlled by changing the focal position of the laser in the plasma to achieve the highest intensity in the plasma in order to maximize the RIT effect. The transmittance, the plasma channel shape in the underdense plasma and the shape of the electron spectrum strongly depend on the focal position of the laser.

Comparison between the shape of the plasma channel and numerical calculations suggests that the laser pulse self-focuses and increases the intensity at an optimum focal position at which the spot diameter is smaller than the plasma wavelength at $n_{\mathrm{c}} / 4$ as a consequence of RSF. The laser transmittance is maximized at this optimal focal position which is expected for superpenetration. A simple estimation of laser energy transmittance which could be used to heat the imploded fuel core by RIT is also shown. 


\section{Optimal focal position for RSF}

In this section, we try to find an optimal focal position of laser pulses for RSF in an inhomogeneous plasma using the paraxial approximation for laser light propagation in the plasma. Two inhomogeneities along the laser propagation axis are treated; one is density inhomogeneity of the plasma and the other is laser spot diameter inhomogeneity due to the convergence of the laser pulse. Unlike in uniform density plasmas, in the FI scheme the laser pulse propagates through increasing density along its path and hence $P_{\mathrm{RSF}}$ decreases with propagation in the underdense plasma. This indicates that the RSF focusing effect and guiding depend on the location of the pulse in the plasma. The laser spot diameter decreases with propagation since the pulse is focused by optics such as parabolic mirrors. If the spot diameter is larger than the plasma wavelength, the beam might break into many filaments [13]. These two inhomogeneities make propagation complex but may allow some room for optimization. In order to obtain insight on laser propagation of converging laser pulses in inhomogeneous plasma, we performed numerical calculations based on the paraxial approximation. The wave equation is a non-linear Schrodinger [7] equation given by

$$
k \frac{\partial a}{\partial z}=\frac{i}{2}\left(\frac{\partial^{2}}{\partial r^{2}}+\frac{1}{r} \frac{\partial}{\partial r}-k_{\mathrm{p}}^{2} \frac{n_{\mathrm{e}}}{n_{0}} \frac{1}{\gamma}\right) a .
$$

Here, $a, k, k_{\mathrm{p}}$ and $n_{0}$ are the normalized vector potential of the laser, the wavenumber of the laser, the wavenumber of the plasma given by $\omega_{\mathrm{p}} / c$ and background plasma density, respectively. The plasma frequency is given by $\omega_{\mathrm{p}}=\sqrt{4 \pi n_{0} e^{2} / m}$. With cylindrical symmetry, the electron density $n_{\mathrm{e}}$ and Lorenz factor for linearly polarized laser light are given by:

$$
\begin{aligned}
& \gamma=\sqrt{1+\frac{|a|^{2}}{2}}, \\
& n_{\mathrm{e}}=\max \left(0, n_{0}+\nabla_{\perp}^{2} \gamma\right) .
\end{aligned}
$$

Negative electron density resulting from the ponderomotive cavitation was treated as zero density. This equation is valid as long as inequalities of $Z_{\mathrm{R}}, L, \gg \lambda_{\mathrm{p}} \gg \lambda$ and $r_{0} \gg \lambda$ hold. $Z_{\mathrm{R}}=\pi r_{0}^{2} / \lambda$ is the Raleigh range, $L$ is the pulse length in the $z$ direction, $r_{0}$ is the $1 / e^{2}$ spot radius of the laser, and $\lambda_{\mathrm{p}}$ and $\lambda$ are plasma wavelength and laser wavelength, respectively. Small inhomogeneities within the density varying in the $z$ direction can be introduced as long as the scale length is much larger than the plasma wavelength. Equation (1) was solved by a semiimplicit finite differential method called the Crank Nicolson scheme [14]. Numerical steps for the spatial grid are approximately $0.0393 \lambda$ and $0.125 \lambda$ for $z$ and $r$ directions, respectively. The $f$-number of the focusing optic, laser power and wavelength of the laser $\lambda$ are fixed at 3.9, $10 \mathrm{TW}$ and $1.053 \mu \mathrm{m}$, respectively. The plasma density profile was set at an exponential function with the scale length of $22 \mu \mathrm{m}$. These parameters are the same as in the experiment described in the next section. No qualitative change was observed when the power was changed to 5 or $15 \mathrm{TW}$.

The results are shown in figure 2 as a function of the focal position of the laser, with a trajectory of the beam radius (half-max of peak vacuum intensity), given by the white dashed line.

Figure 2(a) shows an initial electron density profile along the laser propagation axis. The location of the maximum intensity varies by changing the focal position. If the laser is focused in a low density region as shown in figures $2(b)$ and $(c)$, the beam divergence becomes significant and shows ring structures at the right boundary in figure 2(c). In cylindrical geometry, the ring beam corresponds to laser filamentation. The laser may break into small filaments in the actual 3D case as shown by a back reflected image and a 3D PIC simulation [15]. 


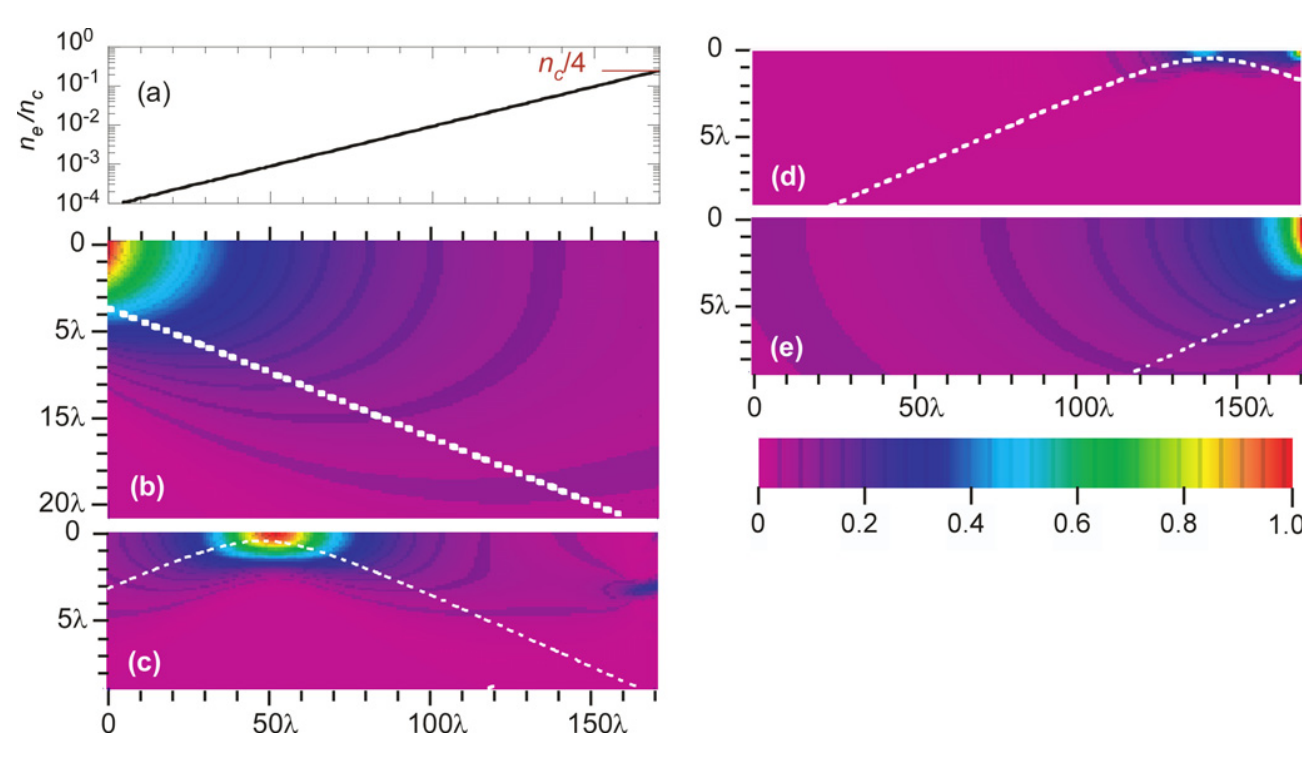

Figure 2. Intensity contour obtained by paraxial calculations. (a) Initial density profile along laser propagation axis. Focal positions in the vacuum are $(b)-47 \lambda,(c) 47 \lambda,(d) 142 \lambda$ and (e) $237 \lambda$, respectively. White dashed lines are the trajectory of the spot radius (half intensity of the peak) for vacuum focus. The laser propagates from left to right.

Therefore, focusing the laser far from the target makes the beam break into filaments. A single collimated beam extending to the right end of the calculation box is observed in figure $2(d)$. Of all the focal positions in figure 2, the spot diameter is smallest (highest intensity) for figure $2(d)$. If the laser is focused further into the plasma as shown in figure 2(e), the beam keeps converging but does not show rapid focusing compared with the vacuum spot diameter. As we showed in figure 1 , a smaller spot diameter at $n_{\mathrm{c}} / 4$ shows fewer filaments due to filamentation instability. The transverse separation of each filament was on the order of the plasma wavelength $\left(\lambda_{\mathrm{p}}\right)$ which equals $2 \lambda$. The spot diameter at $n_{\mathrm{c}} / 4$ (right edge of the calculation box) is smaller than $2 \lambda$ for the optimal condition shown in figure $2(d)$, while it is larger than the $\lambda_{\mathrm{p}}$ for all other focal positions. Therefore, the beam can be collimated via RSF at the optimum focal position but not for other positions. We propose a scenario that the laser spot diameter should be smaller than the plasma wavelength at $n_{\mathrm{c}} / 4$ for whole beam self-focusing. The experimental data shown in the next section such as transmittance measurements and electron energy spectra support this scenario.

We briefly discuss the validation of using the paraxial approximation for the laser light propagation in plasmas. We considered three effects which might affect our results. The first is the effect due to the second derivative of $z$ which is dropped in equation (1). If laser pulse self-focuses down to its wavelength, non-paraxial approximation might be required [16, 17]. This effect is important for propagation only after the focal position. The non-paraxial waveequation is given by [16]

$$
\begin{aligned}
& \frac{k}{c} \frac{\partial a}{\partial t}=\frac{i}{2}\left(\frac{\partial^{2}}{\partial r^{2}}+\frac{1}{r} \frac{\partial}{\partial r}+\frac{\partial^{2}}{\partial z^{2}}-k^{2} n^{2}\right) a, \\
& n^{2}=1-\left(\frac{\omega_{\mathrm{p}}}{\omega}\right)^{2} \frac{1+k_{\mathrm{p}}^{-2} \Delta \gamma}{\gamma} .
\end{aligned}
$$

The difference between equations (1) and (3) is the second derivative of $z$ on the right-hand side of equation (3). The term increases diffraction loss since a wide angle of rays is taken into 


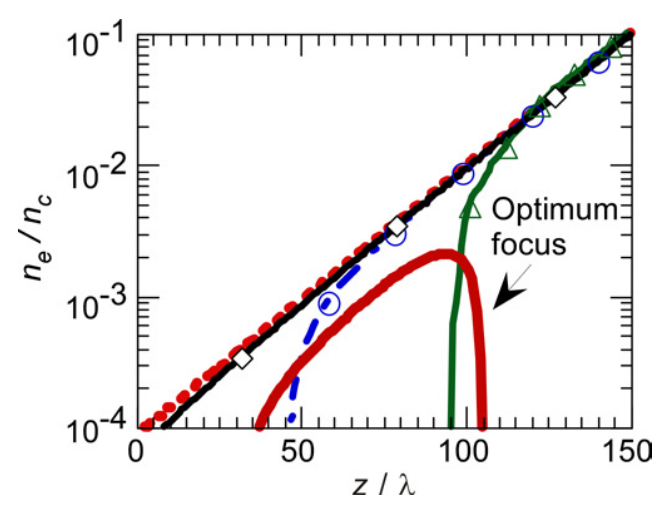

Figure 3. Longitudinal electron density profile at radius $r=0.625 \lambda$ in the channel as obtained by calculations (color online). The focal positions are at $-47 \lambda$ (dash-dotted line with circles), $47 \lambda$ (solid line with triangles), $142 \lambda$ (solid line) and $237 \lambda$ (solid line with diamonds), respectively. The initial electron density profile is shown by a dashed line for comparison. The laser propagates from left to right.

account in equation (3). Consequently, intensity enhancement is reduced due to diffraction, and an off axis channel could be formed [16]. This means that the laser pulse may break into filaments after the smallest spot diameter in figure 2. Despite this effect, the paraxial approximation might be valid for gradual focusing before the spot diameter reduces down to the wavelength of the laser. Gradual focusing cases are shown in figures $2(b)$ and $(e)$. Therefore, focusing the laser far from or close to the plasma does not enhance laser intensity significantly and there will be an optimal focal position for intensity enhancement. Simulation based on more rigorous models such as equation (3) and 3D PIC codes are desirable in order to study laser propagation at the optimum focus condition. Experimental observations shown in the next section confirm that the optimal focal position is the position obtained by the calculations using the paraxial approximation. Therefore, filamentation for the optimal focal position should be less severe than for other focal positions.

The second effect is the excitation of plasma waves and feedback to the laser pulse from the plasma waves, such as SRS. Large angle scattering can cause significant energy loss due to SRS $[18,19]$ and lower the transmission of the laser. SRS growth could be reduced by density reduction in the plasma channel since the growth rate is proportional to electron density [20]. Plasma density in the plasma channel is different for different focal positions since density reduction is large where laser intensity is large. The on-axis density profile is shown in figure 3 as a function of focal position from our calculations. The longitudinal density profiles in the calculations were extracted at a fixed radius $r=0.625 \lambda$. The on-axis electron density is minimized for the optimal focal position. The SRS is less severe at the optimal position but more severe for others. Therefore less energy loss due to the SRS is expected for the optimal position.

The third effect is the acceleration of the electron beam and feedback to laser propagation. The relativistic electron can drag the laser close to or beyond the critical density as shown by Pukhov et al by 3D PIC simulations [10] and is consistent with experiments [21], since electrons move faster than light in plasmas. The space charge of the electron beam expels the plasma electrons and forms a plasma channel, and then the light is guided by the channel. This mechanism works where the relativistic electron beam density is comparable to the background density. Therefore, if the beam density is comparable to the peak density of the experiments 23-50 $n_{\mathrm{c}}$, this mechanism might be effective. 

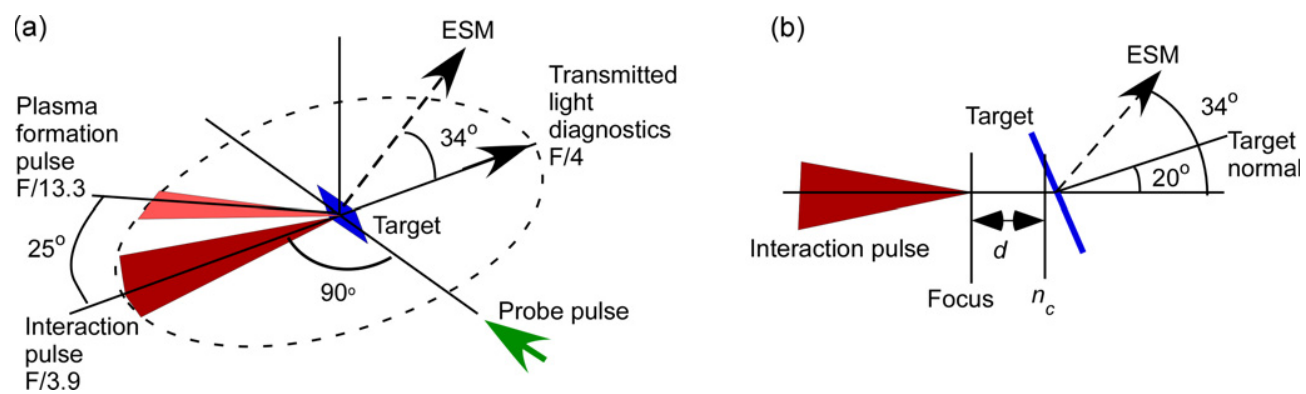

Figure 4. The experimental configuration with $(a)$ air view and $(b)$ side view. Both the laser axis and the diagnostics axis are in the same plane except for the electron spectrometer (ESM). The probe laser axis is normal to the interaction pulse and parallel to the target surface. The target normal axis is tilted $20^{\circ}$ down from the interaction pulse axis as shown in $(b)$. The focal position parameter $d$ is shown in $(b)$

\section{Focus optimization experiment on the 20 TW GEKKO module II (GMII) laser system}

The scenario in the previous section was examined by the Gekko Module II (GMII) 20 TW glass laser system at the Institute of Laser Engineering of Osaka University [22]. The experimental configuration is shown in figure 4. A plasma formation pulse of $1.053 \mu \mathrm{m}$ wavelength was focused by an $F / 13.3$ lens onto a thin deuterated plastic foil with a $0.1 \mu \mathrm{m}$ thick Al coating. The pulse had a Gaussian temporal shape with 400 ps width at half maximum (FWHM) duration with a peak intensity of $(3-5) \times 10^{13} \mathrm{~W} \mathrm{~cm}^{-2}$ with $1.6-3.3 \mathrm{~J}$ of energy. The thickness of the plastic layer was varied to change the peak density of the plasma from 23 to $75 n_{\mathrm{c}}$ for corresponding thicknesses of 0.25 to $1 \mu \mathrm{m}$. The plasma density profile was calculated by the ILESTA-1D hydrodynamics code [23]. The density profile obtained by the code had been experimentally examined up to $30 n_{\mathrm{c}}$ by measurement with x-ray laser grid image reflectometry (XRL-GIR) [24] and by optical laser probe interferometry [25]. The density profile in the underdense plasma did not depend on the thickness of the foil. After a 40 ps delay from the peak of the plasma formation pulse, an interaction laser pulse with $600 \mathrm{fs}$ duration was focused by an F/3.9 off axis parboiled mirror to the plasma at a $20^{\circ}$ incidence angle with $\mathrm{S}$ polarization. The peak power and wavelength of the pulses were $11-16 \mathrm{TW}$ and $1.053 \mu \mathrm{m}$, respectively. The focal spot was measured by an imaging system with an $F / 2$ collection lens using the regenerative amplifier without firing the glass amplifiers. The spot was elliptical with $13 \mu \mathrm{m}$ and $33 \mu \mathrm{m}$ FWHM for the horizontal and the vertical widths, respectively. The FWHM region contained $50 \%$ of the spot energy. Note that the probe laser axis was in the horizontal plane. The focused intensity is calculated to be $(1.6-2.4) \times 10^{18} \mathrm{~W} \mathrm{~cm}^{-2}$. The Lorenz factor for the intensity range is between 1.3 and 1.4. Therefore, the interaction pulse should be reflected at approximately $n_{\mathrm{c}}$ without intensity enhancement due to RSF. All laser pulses were seeded by the same oscillator resulting in perfect synchronization among three pulses (heating, interaction and probe).

Three diagnostics were used to study the propagation: a transverse optical interferometer, a laser transmittance measurement and an energy spectrum of the relativistic electrons. A fraction of the laser pulse was sent to a grating compressor to compress down to $20 \mathrm{ps} \mathrm{FWHM}$ and was frequency doubled by a KDP (potassium dihydrogen phosphate) crystal as a probe beam. The probe timing was synchronized with the arrival of the interaction pulse at the target within $20 \mathrm{ps}$. The probe pulse of $20 \mathrm{ps}$ duration is too long to resolve the fine scale of the channel due to channel expansion after the interaction pulse. The plasma is imaged 


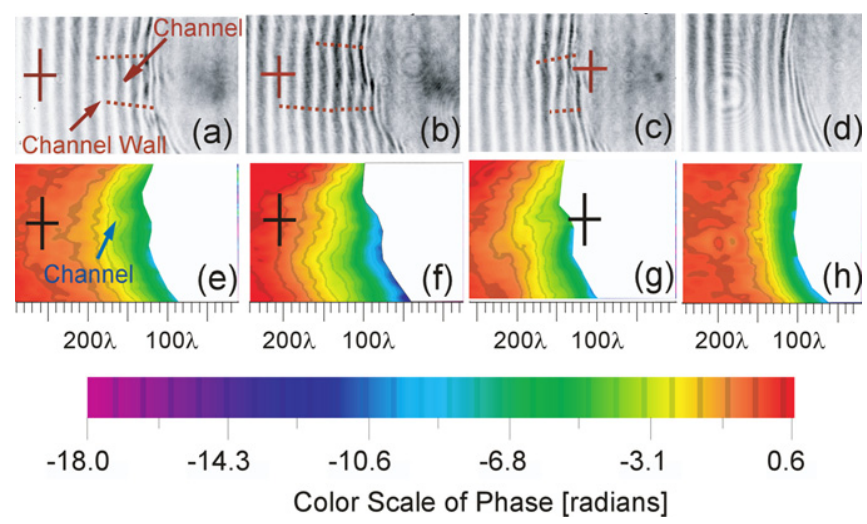

Figure 5. Gray scale image of interferogram and phase contour image. The focal positions are $(a)$, $(e) d=300,(b),(f) d=200$ and $(c),(g) d=100$, respectively. The crosses indicate the focal position in vacuum. The laser propagates from left to right. $(d)$ and $(h)$ are images without the interaction pulse. The location of the plasma channel wall is indicated by dashed lines in figures $(a),(b)$ and $(c)$.

by the probe onto a modified Normarski interferometer using a Fresnel prism [25] with a spatial resolution better than $10 \mu \mathrm{m}$. Transmitted light was collected by an F/4 optic and the target plane was imaged onto a charge coupled device (CCD) camera through an interference filter centered at $1.053 \mu \mathrm{m}$ wavelength with a $10 \mathrm{~nm}$ bandwidth. The temporal waveform was monitored through an identical interference filter by a Hamamatsu S1 Streak Camera after taking a reflection from a wedge inserted in front of the CCD camera. The temporal resolution was $50 \mathrm{ps}$. The CCD signal is absolutely calibrated to energy with a calorimeter with laser shots into vacuum. No pre-pulses were observed before the main pulse within a $10^{6} \mathrm{~S} / \mathrm{N}$ ratio.

The transmitted signal at $1.053 \mu \mathrm{m}$ may have stray light originating from the plasma formation pulse because the two pulses are at the same wavelength. This stray light could mix into the transmitted measurement. The transmittance of the plasma formation pulse through the plasma channel formed by the interaction pulse was simulated by $2 \mathrm{D}$ ray tracing calculations. Up to $2 \%$ of the transmittance could be due to the plasma formation pulse. In the calculation, a parabolic radial density profile and a uniform longitudinal density were assumed. The radial expansion of the channel was taken into account at a constant velocity of $10^{8} \mathrm{~cm} \mathrm{~s}^{-1}$ which was confirmed by transverse interferometry by changing the delay of the probe pulse and is consistent with other experiments [26-29]. The initial diameter of the channel was set at $10 \mu \mathrm{m}$. The density at the edge of the channel was assumed to be the critical density. All the rays of the plasma formation pulse inside the channel were traced. The angle between the plasma formation pulse axis and the axis of the channel was $25^{\circ}$ corresponding to the incidence angle of the plasma formation pulse. The length of the channel was set at $200 \mu \mathrm{m}$ including the underdense and overdense regions. The energy of rays contained within the solid angle of the collection optics $(F / 4)$ was integrated. The calculated value of the transmitted energy was $140 \mathrm{~mJ}$. This means that up to $2 \%$ of laser energy could mix into the transmission measurement. We consider that this noise source may be much smaller in the experiment due to scattering via plasma fluctuation.

We introduce a parameter ' $d$ ' which indicates the distance in microns between the focal position of the interaction pulse and the critical density point as shown in figure $4(b)$. Interferograms with inferred phase images are shown in figure 5 as a function of ' $d$ '. 
The phase contours correspond to the relative electron density, since the cylindrical symmetry is not good and thus Abel inversion is no longer valid. The electron density between $0.01 n_{\mathrm{c}}$ and $0.05 n_{\mathrm{c}}$ is obtained in the interferograms when we set a cylindrical symmetry condition to the axis of the interaction pulse. The maximum density is limited by refraction due to the density gradient and is approximately $0.1 n_{\mathrm{c}}$ for a spherically symmetric plasma of density scale length between 10 and $100 \mu \mathrm{m}$. The discrepancy of the estimation from the experiment could be due to the non-spherical shape of the plasma. The minimum density is determined by defining the zero phase shift contour in the phase image. The possible error is less than $0.5 \pi$ radian in phase. The estimated error by assuming a uniform density of $200 \mu \mathrm{m}$ length along the probe axis is on the order of $0.01 n_{\mathrm{c}}$.

The interaction pulse propagates from the left to the right in the figures and its focal position is varied along its axis. Plasma channels formed by the interaction pulse appear in the region where the contours bend toward the right in the phase images. The positions of the channel walls (dashed line) are shown in figures $5(a)-(c)$. The difference between images with (figures 5(a)-(c)) and without the interaction pulse (figure $5(d)$ ) is clear. When the interaction pulse was focused at $d=300$ (figures 5(a), $(e)$ ), a channel is seen at 100 $\lambda$ away from the focus but not in the vicinity of the focus. Remember that the calculation shows weak refocus and ring focus formation after $100 \lambda$ away from the focus (the corresponding calculation is figure 2(c)). The plasma channel could be due to this refocusing effect. The fact that no channel is seen in the vicinity of the focus indicates that the phase shift due to the channel is too small to detect. Two factors can be considered. One is that the density depression of the channel is too small. The other is that its radius is too small. Both effects are due to the low laser intensity in the corresponding region. The typical radial channel expansion speed observed was $10^{8} \mathrm{~cm} \mathrm{~s}^{-1}$ which was confirmed by transverse interferometry by changing the delay of the probe pulse and is consistent with other experiments [26-29]. The plasma channel formed by the interaction pulse will have a larger diameter than the size at its formation if the laser intensity is sufficiently high enough to drive radial expansion. For the smaller channel diameter, a low laser intensity could possibly be due to energy scattering via SRS. When the laser was focused at $d=200$ (figures $5(b)$ and $(f)$ ), a plasma channel is seen continuously from $80 \lambda$ up to $200 \lambda$. This is different from the case when the focal position was at $d=300$. The channel diameter of about $80 \lambda$ is much larger than the simulated one shown in figure $2(d)$ because of the relatively long probe pulse duration. When the laser was focused at $d=100$ (figures $5(c)$ and $(g)$ ), a plasma channel is seen at around $150 \lambda$. The smaller diameter of the channel compared with $d=200$ suggests that the laser intensity is weaker in this case. It is possible that the focus is set too deep into the plasma to enhance intensity due to RSF. As shown in the corresponding calculation results in figure $2(e)$, the distance might be too short for RSF to enhance the intensity.

Therefore, by comparing the location and length of the channel in three different focal positions, we conclude that the plasma channel moved by changing the focal position of the laser. The largest channel diameter occurs at $d=200$ suggesting that this is optimum for RSF corresponding to the optimum condition shown by the calculation (figure $2(d)$ ).

The transmittance depends strongly on the focal position and shows a maximum when the interaction pulse was focused at $d=200$ as shown in figure 6(a). This focal position is consistent with the optimum condition for channel formation obtained by the interferogram. The error bar for the transmittance is due to the noise in the diagnostics. The error bar for the density in figure $6(b)$ is due to the energy fluctuation of the plasma formation pulse. The peak density of the plasma $\left(50 n_{\mathrm{c}}\right)$ could be too large for a larger transmitted value. When the preformed plasma density was reduced while keeping the focus at $d=200$, the transmittance increases as shown in figure $6(b)$. The transmittance of approximately $0.03 \%$ was obtained 

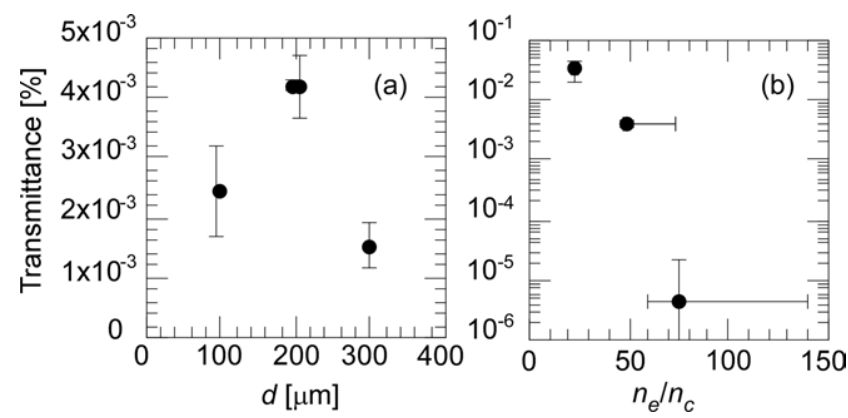

Figure 6. Transmittance as a function of (a) focal position at peak plasma density of $50 n_{\mathrm{c}}$ and of (b) the peak density in preformed plasma at $d=200$, respectively. An explanation for the error bars is in the text.

when the peak density was $23 n_{\mathrm{c}}$. Figure $6(a)$ indicates a clear dependence on the laser focal position even including the experimental error bars suggesting that stray light from plasma formation beam effect is negligibly small.

This transmission could be related to how the laser propagates through the overdense plasma. Sentoku et al showed in a 3D PIC simulation that a $6.25 \times 10^{20} \mathrm{~W} \mathrm{~cm}^{-2}$ laser pulse can penetrate up to $20 n_{\mathrm{c}}$ density without diffraction [30]. The density profile of the simulation is identical to our experiments with a peak density of $50 n_{\mathrm{c}}$. The pulse front velocity $\left(v_{\mathrm{f}}\right)$ decelerates during penetration because the plasma thermal pressure is strong enough to slow the laser pulse. The laser can penetrate a distance which is given by $v_{\mathrm{f}} \tau$ within the duration of the laser $\tau$. The transmittance can be estimated by $\left(\tau-l / v_{\mathrm{f}}\right) / \tau$ for uniform plasmas. Here $l$ is the thickness of the overdense layer. Therefore, larger $v_{\mathrm{f}}$ yields larger transmittance. If our laser self-focuses down to a $2 \lambda$ diameter at the critical density, the intensity becomes $3 \times 10^{20} \mathrm{~W} \mathrm{~cm}^{-2}$. The paraxial calculation actually gives a peak intensity of $8.2 \times 10^{20} \mathrm{~W} \mathrm{~cm}^{-2}$ at $n_{\mathrm{c}} / 4$ for the optimum condition (figure 2(d)). By using Sentoku's HB speed (equation (24) in [30]), the intensity of $1.2 \times 10^{21} \mathrm{~W} \mathrm{~cm}^{-2}$ for the density of $23 n_{\mathrm{c}}$ is required in order to have a positive value of $v_{\mathrm{f}}^{2}$. Intensity below this value gives a negative value for $v_{\mathrm{f}}^{2}$ which means that propagation is impossible since the plasma pressure exceeds the photon pressure of the interaction pulse.

The $v_{\mathrm{f}}$ was calculated for the density profile with the lowest peak density in the experiments. The velocity and the density profile are plotted in figure 7 . The density profile is obtained from the ILESTA-1D simulation and underdense plasma density is neglected in the calculation. We assumed a flattop waveform of the incident pulse at an intensity of $1.2 \times 10^{21} \mathrm{~W} \mathrm{~cm}^{-2}$. The time required for penetrating the overdense plasma is $447 \mathrm{fs}$. The estimated value is close to the FWHM of our laser pulse ( $600 \mathrm{fs})$. Therefore, the transmittance could become very low. The $v_{\mathrm{f}}$ decreases as the density increase and approaches zero at around the peak density. The dense region around the density peak slows down the pulse and restricts the transmission. The transmittance mostly depends on the thickness and the peak density at fixed laser intensity. The estimation tells us that the intensity must be at least $10^{21} \mathrm{~W} \mathrm{~cm}{ }^{-2}$ in order to see any transmittance. This intensity might be too high to explain transmitted light which was observed in our experiments. The estimated intensity from our numerical calculation is $8.2 \times 10^{20} \mathrm{~W} \mathrm{~cm}^{-2}$, which is lower than this estimate for the required intensity for transmission. The required intensity can be relaxed by plasma expansion via heating due to the laser pulse as suggested by Fuchs et al [1]. The pulse front velocity increases with decrease in the electron density. Therefore, transmission can be expected even for laser intensity below 


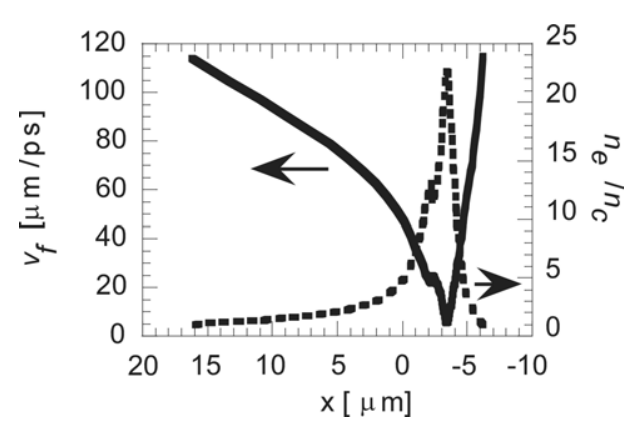

Figure 7. HB velocity. The solid line is the velocity and the dashed line is the assumed density profile. The velocity is calculated from equation (24) in [30] (details are given in the text). The density profile is obtained from the ILESTA-1D simulation at $0.25 \mu \mathrm{m}$ of foil thickness. The laser propagates from left to right.

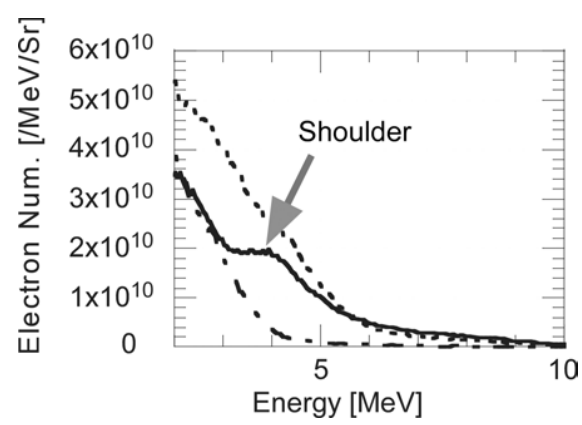

Figure 8. Electron spectra as a function of focal position. The dashed, solid and dash-dotted lines are for the focal position at $d=100,200$ and 300, respectively.

the required value if the electron density decreases from the initial value during the laser pulse due to expansion of the plasma.

The distance between the quarter critical and the critical density is approximately $30 \mu \mathrm{m}$ and is comparable to the Raleigh length $\left(Z_{\mathrm{R}}=20 \mu \mathrm{m}\right)$ for the FWHM spot diameter of $2 \lambda$. The consistency between the optimum condition for the transmittance and the matching condition for the RSF shown by the paraxial calculation suggests that the laser most likely propagated without significant diffraction in this region. Therefore, transmission data support our scenario for the optimum focal condition.

The energy spectra of the electrons were measured by a magnetic spectrometer placed at $34^{\circ}$ from the interaction pulse axis as shown in figure 4. Electrons escaping from the targets were collected through a pinhole having a solid angle of $1.28 \times 10^{-5}$ sr. Imaging plates (IP) were used to record electron signals which were absolutely calibrated by a linear accelerator up to $100 \mathrm{MeV}$. Detailed information about the IP is in [31].

Measured electron spectra as a function of the focal position are shown in figure 8. Two spectral components are shown clearly, one of which is a shoulder at $4 \mathrm{MeV}$ and the other smoothly decaying curves (background). The shoulder appears when $d=200$, which is the optimum condition for RSF according to the interferogram and transmittance. The shoulder is sensitive to the focal position and disappears when the position moved by $100 \mu \mathrm{m}$ from the optimum value. This suggests that electrons in the shoulder are generated and accelerated in the plasma channel. A possible mechanism is betatron acceleration in the plasma channel [32]. On 
the other hand, the electrons in the background spectra are observed in all three focal positions $(d=100,200,300)$. The background electrons could be generated from interaction between filamented laser beams and the underdense plasma. The background electrons could also be generated from the critical density where the laser absorption might be large. However, the fact that the background electrons were observed at $d=300$ might exclude this explanation. According to the interferogram and calculations shown in section 2 , the interaction pulse diverges in underdense plasma and the intensity at the critical density is expected to be low. The laser intensity might be too low to generate electrons at the critical density. The appearance of the shoulder in the electron spectrum at the optimum focal position is consistent with selffocusing of the laser in the plasma and with results obtained by the interferograms and the transmittance measurements. For the experimental data point at $d=200$, which is predicted by the paraxial approximation calculation to be the optimum position for super-penetration, all three diagnostics indicated that penetration was maximized. Therefore our scenario that the spot diameter of the laser pulse at $n_{\mathrm{c}} / 4$ should be smaller than the plasma wavelength in order to achieve whole beam self-focusing, was confirmed by experiments as well as the calculations.

\section{Effectiveness of super-penetration for FI}

The electron energy spectra shown in figure 8 indicate the greatest number of electrons at $2 \mathrm{MeV}$ for $d=100$, which is considered to be the best energy for core heating in FI [4,33]. However, two concerns prevent us from concluding that $d=100$ is the optimum condition for the core heating. First, careful analysis is required for the number of electrons escaping from the target. The electron spectrum was observed at an off axis angle which might be close to the edge of the electron beam. The observed electron number is underestimated when the electron beam is collimated. Additionally, the space charge field and the self-generated magnetic field of the electron beam limit the electron number which could escape from the target [34]. Angular distribution measurements of the electron beam are essential in order to address this issue. Second, the heating mechanism of the core by electron beams is still under debate, in particular for the role of Weibel instability [33, 35-37].

Therefore, we focus on laser propagation for FI. In this section, improvement of energy transport for the FI scheme by the use of super-penetration is discussed. As explained in section 1 , the propagation distance and divergence angle of the electron beam determine heating energy. We show how much laser energy could reach the vicinity of the core, i.e. $10 n_{\mathrm{c}}$ for given laser power and duration. Here, the density of $10 n_{\mathrm{c}}$ was chosen since penetration was confirmed up to $10 n_{\mathrm{c}}$ by the experiments of this paper. Note that the $n_{\mathrm{c}}$ is ciritical density for laser pulse of $\lambda=1.053 \mu \mathrm{m}$.

In order to model laser propagation, a 1D exponential electron density profile is assumed, i.e. $n_{\mathrm{e}}(z)=n_{\mathrm{c}} \exp \left(z / L_{\mathrm{s}}\right)$ with scale length of $L_{\mathrm{s}}=163.8 \mu \mathrm{m}$. The scale length is obtained by a curve fitting in the density range between $n_{\mathrm{c}}$ and $10 n_{\mathrm{c}}$ to the simulated density profile obtained from ILESTA-1D [23] for the FIREX II project [38] of the Institute of Laser Engineering, Osaka University. The peak density at the maximum fuel compression time is $288 \mathrm{~g} \mathrm{~cm}^{-3}$ and the location of the peak is at $27 \mu \mathrm{m}$ from the center of the core. $10.8 \mathrm{~kJ}$ fusion energy is released in the form of $\alpha$ particles when $1 \%$ of the fuel mass (DT) was burned by fusion reaction. The present design of FIREX II (fast ignition realization experiment) allows fusion energy gain close to unity $(Q \sim 1)$. The distance between $n_{\mathrm{c}}\left(10 n_{\mathrm{c}}\right)$ and the peak density point is $573 \mu \mathrm{m}(195 \mu \mathrm{m})$, as shown in figure $9(d)$. A flat top temporal shape laser pulse with a duration of $\tau=10 \mathrm{ps}$ at a power of $P=1 \mathrm{PW}$ was assumed to propagate in the $+z$ direction. The energy transmittance of the laser pulse ( $\left.T_{\text {laser }}\right)$ which propagates from $n_{\mathrm{c}}$ to $10 n_{\mathrm{c}}$ is given 
by the equation:

$$
\begin{aligned}
\tau_{\text {pen }} & =\int_{0}^{z_{0}} \frac{1}{v\left(n_{\mathrm{e}}(z)\right)} \mathrm{d} z, \\
T_{\text {laser }} & =\frac{\tau-\tau_{\text {pen }}}{\tau} .
\end{aligned}
$$

Here, $\tau_{\text {pen }}$ is the time required for the laser pulse front to reach the $10 n_{\mathrm{c}}$ density point at $z_{0}$. The laser pulse front velocity $v$ is calculated by equations (6) and (7), which corresponds to the laser group velocity in relativistic limit. Equation (7) takes into account absorption of the laser pulse and showed a best fit with 3D PIC simulation results up to $10 n_{\mathrm{c}}$ [30]:

$$
\begin{aligned}
& v_{\mathrm{g} 1}\left(n_{\mathrm{e}}\right)=c \sqrt{1-\frac{n_{\mathrm{e}}}{\gamma n_{\mathrm{c} \lambda}}}, \\
& \gamma=\sqrt{1+C_{\mathrm{pol}} a^{2}}, \\
& v_{\mathrm{g} 2}\left(n_{\mathrm{e}}\right)=c \alpha \sqrt{1-\frac{n_{\mathrm{e}}}{\gamma_{\mathrm{OS}} n_{\mathrm{c} \lambda}}}, \\
& \gamma_{\mathrm{OS}}=\sqrt{1+\alpha C_{\mathrm{pol}} \frac{\left(a+a_{\mathrm{c}}\right)^{2}}{4}}, \\
& a_{\mathrm{c}}=\sqrt{\frac{1}{C_{\mathrm{pol}}}\left\{\left(\frac{n_{\mathrm{e}}}{n_{\mathrm{c} \lambda}}\right)^{2}-1\right\}} .
\end{aligned}
$$

Here, $n_{\mathrm{c} \lambda}$ is the critical density for the laser pulse at a wavelength of $\lambda$. $C_{\mathrm{pol}}$ accounts for differences of laser polarization, i.e. 0.5 for linear and 1 for circular polarizations. $\alpha$ is a cycle number measured in units of laser cycles in which the laser pulse amplitude drops from the initial value to a critical value $a_{\mathrm{c}}$ which fulfills the RIT condition $n_{\mathrm{e}}=\gamma n_{\mathrm{c}}$.

As shown in equation (5), the greater pulse front velocity gives the greater transmittance and therefore larger heating energy. The velocity estimated by equations (6) and (7) together with three different HB velocities which assume momentum balance between the plasma and the laser pulse, i.e. Wilks's scaling [39], Sentoku's scaling from equation (24) in [30] and Ping's scaling [40], are shown in figures $9(a)-(c)$ in the density range between $n_{\mathrm{c}}$ and $10 n_{\mathrm{c}}$. Note that 'standard' Wilks's scaling is approximately the same as Sentoku's scaling. Wilks's scaling had been updated by Ping et al by taking into account relativistic mass correction of electrons and had been confirmed by experiments in which Doppler shift was measured in order to infer the motion of the critical surface into solid targets [40]. All three HB velocities are much smaller than that of RIT in the parameter range as shown in figure 9. Therefore, the HB does not contribute energy transmittance of the laser in this discussion and neglecting this effect for simplicity is valid.

The transmittance of the laser pulse could reach more than $75 \%$ once the laser intensity exceeds RIT threshold, determined by the condition $n_{\mathrm{e}}<\gamma n_{\mathrm{c}}$, as shown in figure $9(e)$. On the other hand, no transmittance is expected for the intensity less than the threshold of $3 \times 10^{20} \mathrm{~W} \mathrm{~cm}^{-2}$, which can be achieved by focusing PW laser pulse to a spot diameter of 2 $r_{0}=21 \mu \mathrm{m}$. The Rayleigh length for the spot diameter is $Z_{\mathrm{R}}=960 \mu \mathrm{m}$ and is greater than the scale length of the plasma considered here. Therefore, if the filamentation instability does not diffract the laser pulse from the pass toward the core, heating should be very efficient. However, the spot diameter of $21 \mu \mathrm{m}$ is 10 times larger than the plasma wavelength at $n_{\mathrm{c}} / 4$; therefore, the beam breaks into filaments as shown by figure 1. Our results suggest that the spot diameter should be smaller than the plasma wavelength $\lambda_{\mathrm{p}}=2 \lambda$ at $n_{\mathrm{c}} / 4$. $2 \lambda$ spot diameter at 

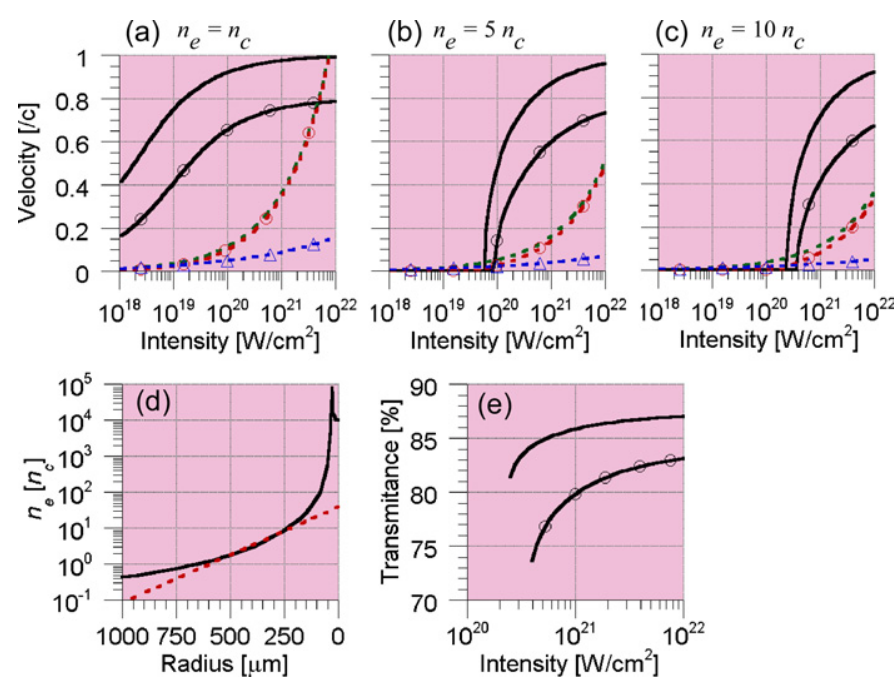

Figure 9. Pulse front velocity at the density of $(a) n_{\mathrm{c}},(b) 5 n_{\mathrm{c}}$ and (c) $10 n_{\mathrm{c}}$ as a function of laser intensity. Electron density profile from ILESTA-1D as a solid line and the fitting curve is shown as the dashed line in $(d)$. The laser transmittance through $n_{\mathrm{c}}$ to $10 n_{\mathrm{c}}$ is shown in figure $(e)$. Legends representing the different propagation models, i.e. the solid line: RIT equation (6), the solid line with circle: Sentoku's RIT scaling equation (7), the dashed line: Wilks's HB scaling, the dashed line with circle: Sentoku's HB scaling from equation (24) in [30] (details are given in the text of section 3), the dashed line: Ping's HB scaling with relativistic correction. Note that the electron density of figure $(d)$ is normalized by the critical density for $1.053 \mu \mathrm{m}$ wavelength.

the laser power of $\mathrm{PW}$ yields $8 \times 10^{20} \mathrm{~W} \mathrm{~cm}^{-2}$. Super-penetration is still required in order to achieve such a narrow channel. Propagating such a narrow channel for orders of mm distance is a challenging subject and more study has to be done by experiments and simulations.

The intensity might be too high for efficient heating by an electron beam. The electron temperature for the ponderomotive scaling is given by $T_{\text {pon }}=m c^{2}(\gamma-1)$ [39]. As shown in figure 8 , the electron spectrum shows an increase in the electron number at $4 \mathrm{MeV}$, when superpenetration occurs. The ponderomotive temperature scaling for $10^{21} \mathrm{~W} \mathrm{~cm}^{-2}$ laser intensity, which is necessary to explain the laser transmittance of this experiment, yields $9.8 \mathrm{MeV}$ and much higher than $4 \mathrm{MeV}$. This estimation might show that ponderomotive scaling is no longer valid for such high laser intensities and that the electron temperature might be lower than the ponderomotive scaling. A possible mechanism of this temperature lowering is density steepening of the laser plasma interface during the interaction. Temperature lowering by this effect had been observed in PIC simulations [35] and might promote heating efficiency.

There are many issues and difficulties which are not experimentally clear at this moment, such as the heating mechanism due to electron beam, stable propagation of the laser at narrow channel diameters for orders of millimetre distance and electron temperature under density steepening. A shorter wavelength laser pulse might remove all of these issues and difficulties. The $70 \%$ conversion efficiency of the frequency doubling process at $1.053 \mu \mathrm{m}$ had been demonstrated for chirped pulse amplification systems $[41,42]$. By frequency doubling, a number of benefits for FI with super-penetration are available. First, shorter propagation distances due to the increase in the critical density by a factor of 4; second, decreasing laser vector potential $a$ and therefore the electron temperature. Additional reduction of the propagation distance could be available by the use of circular polarization. The Lorentz factor depends on polarization, as shown by equations (6) and (7), and is proportional to the factor $C_{\mathrm{pol}}$ 
at the ultra-relativistic limit $(a \gg 1) . \gamma$ for circular polarization is twice as high as $\gamma$ for linear polarization. This polarization factor relaxes the RIT condition by a factor of 2 without expense of laser energy. We estimated the energy coupling efficiency for $1.053 \mu \mathrm{m}$ and $0.527 \mu \mathrm{m}$ laser pulses to the core by assuming the laser pulse generates an electron beam at $10 n_{\mathrm{c}}\left(40 n_{\mathrm{c}}\right)$ for $1.053 \mu \mathrm{m}(0.527 \mu \mathrm{m})$ and that the electron beam propagates from the generation point to a core of $57 \mu \mathrm{m}$ diameter with a divergence angle of $30^{\circ}$ with straight trajectories. When an identical electron number and electron spectrum are assumed for both wavelengths, calculations show that 4.4 times more electrons could hit the core for the $0.527 \mu \mathrm{m}$ laser pulse than the $1.053 \mu \mathrm{m}$ laser pulse. Heating must be more efficient in the $0.527 \mu \mathrm{m}$ laser pulse compared with the $1.053 \mu \mathrm{m}$ laser pulse since electron temperature should be lower for the $0.527 \mu \mathrm{m}$ laser pulse.

We showed in this section the effectiveness of super-penetration for the FI scheme by considering transmittance of the laser pulse. RIT is the most effective process for energy transfer from critical density to the $10 n_{\mathrm{c}}$ density point due to high pulse front speed. Superpenetration might be required in order to avoid laser beam filamentation for a PW laser pulse since the spot diameter is much larger than the plasma wavelength at $n_{\mathrm{c}} / 4$. However, a narrow channel diameter as a consequence of super-penetration yields high laser intensity which might not be suitable for core heating. An alternative method using a shorter wavelength laser pulse is considered for core heating and it is shown that energy coupling efficiency could be improved by a factor of 4.4 by using a frequency doubled circular polarization laser pulse.

\section{Conclusions}

The focal position of the laser strongly affects the laser propagation in an inhomogeneous plasma. The paraxial approximation indicated different propagation modes depending on the focal position. We introduced a scenario: the laser spot diameter at $n_{\mathrm{c}} / 4$ density must be smaller than the plasma wavelength to achieve whole beam self-focusing. An optimum focus was found experimentally by measuring the plasma channel, transmittance and electron spectra. This optimum focal position was consistent with that predicted by the paraxial approximation calculation. All three data are consistent with one another and show that the laser likely performed RSF most effectively at the optimum focus in which the scenario was satisfied. The instability at $n_{\mathrm{c}} / 4$ is a key for propagation into overdense plasma at relativistic intensities. Energy transmittance of the laser pulse to the core was considered by a 1D propagation model of RIT and showed reasonable energy transfer from critical density to the $10 n_{\mathrm{c}}$ density point in a large scale plasma. When our scenario for super-penetration was applied to the FI experiments (FIREX II), electron temperature was found to be too high for efficient heating via high focused laser intensity. The experimentally observed electron spectrum showed lower electron temperature than the ponderomotive scaling possibly due to density steeping of laser plasma interface. We found that a frequency doubled circular polarization laser pulse potentially improves the laser transmittance for the FI due to greater critical density and lower normalized vector potential.

\section{Acknowledgments}

The authors acknowledge all the technical support of the engineering staff at the Institute of Laser Engineering for the laser system operation, target fabrication and data acquisition.

TM acknowledges the research fellowships support by the Japan Society for Promotion of Science (JSPS). ALL and JZ acknowledge both the postdoc (JSPS) and the Japan/China 
core university programmes supported by JSPS operated by the National Institute of Fusion Science.

\section{References}

[1] Fuchs J et al 1998 Phys. Rev. Lett. 802326

[2] Kodama R, Takahashi K, Tanaka K A, Tsukamoto M, Hashimoto H, Kato Y and Mima K 1996 Phys. Rev. Lett. 774906

[3] Tanaka K A, Hashimoto H, Kodama R, Mima K, Sentoku Y and Takahashi K 1999 Phys. Rev. E 603283

[4] Tabak M, Hammer J, Glinsky M E, Kruer W L, Wilks S C, Woodworth J, Campbell E M, Perry M D and Mason R J 1994 Phys. Plasmas 11626

[5] Kodama R et al 2001 Nature 412798

[6] Willi O, Campbell D H, Schiavi A, Borghesi M, Galimberti M, Gizzi L A, Nazarov W, Mackinnon A J, Pukhov A and Meyer-ter-vehn J 2001 Laser Part. Beams 195

[7] Esarey E, Sprangle P, Krall J and Ting A 1997 IEEE J. Quantum Electron. 331879

[8] Tanaka K A et al 2000 Phys. Plasmas 72014

[9] Kodama R et al 2001 Phys. Plasmas 82268

[10] Pukhov A and Meyer-ter-Vehn J 1996 Phys. Rev. Lett. 763975

[11] Forslund D W, Kindel J M and Lindman E L 1975 Phys. Fluids 181002

[12] Sentoku Y and Kemp A 2008 J. Comput. Phys. 227 6848-61

[13] Feit M D, Komashko A M, Musher S L, Rubenchik A M and Turitsyn S K 1998 Phys. Rev. E 577122

[14] Garcia A L 1994 Numerical Method For Physics (Englewood Cliffs, NJ: Prentice-Hall) p 243

[15] Tanaka K A et al 2000 Phys. Rev. E 622672

[16] Ritchie B 1994 Phys. Rev. E 50 R687

[17] Bolton P R and Ritchie B 1997 J. Opt. Soc. Am. B 14437

[18] Antonsen T M Jr and Mora P 1992 Phys. Rev. Lett. 692204

[19] Monot P et al 2001 Phys. Plasmas 83766

[20] Walton B R et al 2006 Phys. Plasmas 13113103

[21] Lei A et al 2007 Phys. Rev. E 76066403

[22] Kitagawa Y et al 1999 Fusion Eng. Des. 44261

[23] Takabe H et al 1988 Phys. Fluids 312884

[24] Kodama R, Takahashi K, Tanaka K A, Kato Y, Murai K, Weber F, Barbee T W and DaSilva L B 1999 Rev. Sci. Instrum. 70543

[25] Adumi K, Tanaka K A, Matsuoka T, Kurahashi T, Yabuuchi T, Kitagawa Y, Kodama R, Sawai K and Suzuki K 2004 Phys. Plasmas 113721

[26] Fuchs J et al 1998 Phys. Rev. Lett. 801658

[27] Borghesi M, Mackinnon A J, Gaillard R, Willi O, Pukhov A and Meyer-ter-Vehn J 1998 Phys. Rev. Lett. 805137

[28] Mackinnon A J et al 1999 Phys. Plasmas 62185

[29] Krushelnick K, Ting A, Moore C I, Burris H R, Esarey E, Sprangle P and Baine M 1997 Phys. Rev. Lett. 784047

[30] Sentoku Y, Kruer W, Matsuoka M and Pukhov A 2006 Fusion Sci. Technol. 49278

[31] Tanaka K A, Yabuuchi T, Sato T, Kodama R, Kitagawa Y, Takahashi T, Ikeda T, Honda Y and Okuda S 2005 Rev. Sci. Instrum. 76013507

[32] Pukhov A, Sheng Z-M and Meyer-ter-Vehn J 1999 Phys. Plasmas 62847

[33] Solodov A A, Betti R, Delettrez J A and Zhou C D 2007 Phys. Plasmas 14062701

[34] Yabuuchi T et al 2007 Phys. Plasmas 14040706

[35] Chrisman B, Sentoku Y and Kemp A J 2008 Phys. Plasmas 15056309

[36] Campbell R B, Kodama R, Mehlhorn T A, Tanaka K A and Welch D R 2005 Phys. Rev. Lett. 94055001

[37] Kemp A J, Sentoku Y, Sotinkov V and Wilks S C 2006 Phys. Rev. Lett. 97235001

[38] Mima K et al 2007 Eur. Phys. J. D 44259

[39] Wilks S C, Kruer W L, Tabak M and Langdon A B 1992 Phys. Rev. Lett. 691383

[40] Ping Y et al 2008 Phys. Rev. Lett. 100085004

[41] Neely D et al 1999 Laser Part. Beams 17281

[42] Queneuille J, Druon F, Maksimchuk A, Cheriaux G, Mourou G and Nemoto K 2000 Opt. Lett. 25508 